\title{
On the Language of Standard Discrete Planes and Surfaces
}

\author{
Damien Jamet \\ LIRMM, Université Montpellier II, 161 rue Ada, 34392 Montpellier Cedex 5 - France \\ jamet@lirmm.fr
}

\begin{abstract}
Abstact A standard discrete plane is a subset of $\mathbb{Z}^{3}$ verifying the double Diophantine inequality $\mu \leq a x+b y+c z<\mu+\omega$, with $(a, b, c) \neq$ $(0,0,0)$. In the present paper we introduce a generalization of this notion, namely the $(1,1,1)$-discrete surfaces. We first study a combinatorial representation of discrete surfaces as two-dimensional sequences over a three-letter alphabet and show how to use this combinatorial point of view for the recognition problem for these discrete surfaces. We then apply this combinatorial representation to the standard discrete planes and give a first attempt of to generalize the study of the dual space of parameters for the latter [VC00].
\end{abstract}

\section{Introduction}

The works related to discrete lines and planes can be roughly divided in two kinds of approaches. In [And93], É. Andrès introduced the arithmetic discrete planes, as a natural generalization of the arithmetic discrete lines introduced by J.P. Reveillès [Rév91]. Since then, using different approaches, many authors have investigated the recognition problem of discrete planes, that is, " given $\mathcal{V} \subseteq \mathbb{Z}^{3}$ a set of voxels, does there exist a discrete plane containing $\mathcal{V}$ ? 》 (using linear programming [Meg84,PS85,VC00,Buz02], arithmetic structure [DRR96] and Farey series [VC00]). An interesting review of these algorithms can be found in [BCK04].

On the other hand, a wide literature has been devoted to the study of Sturmian words, that is, the infinite words over a binary alphabet which have $n+1$ factors of length $n$ [Lot02]. These words are also equivalently defined as a discrete approximation of a line with irrational slope. Then, many attempts have been investigated to generalize this class of infinite words to two-dimensional words. For instance, in [Vui98,BV00b,ABS04], it is shown that the orbit of an element $\mu \in[0,1[$ under the action of two rotations codes a standard discrete plane. Furthermore, the generating problem of one or two-dimensional words characterizing discrete lines or planes is investigated in [BV00b,Lot02,ABS04,BT04].

Let us now introduce some basic notions and notation used in the present paper. Let $\left\{\overrightarrow{e_{1}}, \overrightarrow{e_{2}}, \overrightarrow{e_{3}}\right\}$ denote the canonical basis of the Euclidean space $\mathbb{R}^{3}$. An element of $\mathbb{Z}^{3}$ is called a voxel. It is usual to represent a voxel $(x, y, z) \in \mathbb{Z}^{3}$ as a unit cube of $\mathbb{R}^{3}$ centered in $(x, y, z)$. Another equivalent representation is to 
consider the unit cube $\left\{\left(x+\lambda_{1}, y+\lambda_{2}, z+\lambda_{3}\right) \mid\left(\lambda_{1}, \lambda_{2}, \lambda_{3}\right) \in[0,1]\right\}$. In the present paper, for clarity issues, we consider the last representation.

Let $(a, b, c, \mu, \omega) \in \mathbb{R}^{5}$. An arithmetic discrete plane with normal vector $(a, b, c)$, with translation parameter $\mu$, and with thickness $\omega$, is the subset of $\mathbb{Z}^{3}$ defined as follows:

$$
\mathfrak{P}(a, b, c, \mu, \omega)=\left\{(x, y, z) \in \mathbb{Z}^{3} \mid \mu \leq a x+b y+c z<\mu+\omega\right\} .
$$

If $\omega=\max \{|a|,|b|,|c|\}$, then $\mathfrak{P}(a, b, c, \mu, \omega)$ is said to be a naive discrete plane. If $\omega=|a|+|b|+|c|$, then $\mathfrak{P}(a, b, c, \mu, \omega)$ is said to be a standard discrete plane. Considering the action of the group of isometries on the set of the discrete planes, we can suppose, with no loss of generality, that $0 \leq a \leq b \leq c$ and $c \neq 0$.

It is well known that the naive discrete planes are functional, that is, if $0 \leq$ $a \leq b \leq c$, the naive discrete plane $\mathfrak{P}(a, b, c, \mu, \max \{|a|,|b|,|c|\})$ is in bijection with the integral points of the plane $z=0$ by the projection map $\pi_{z}: \mathbb{R}^{3} \rightarrow$ $\left\{(x, y, z) \in \mathbb{R}^{3} \mid z=0\right\}$ along the vector $(0,0,1)$. In a similar way, in [ABS04], it is shown that, given the affine orthogonal projection along the vector $(1,1,1)$ onto the plane $x+y+z=0$, namely $\pi: \mathbb{R}^{3} \longrightarrow\left\{(x, y, z) \in \mathbb{R}^{3} \mid x+y+z=0\right\}$, and given $\Gamma=\pi\left(\mathbb{Z}^{2}\right)$, then the restriction $\pi: \mathfrak{P}(a, b, c, \mu,|a|+|b|+|c|) \longrightarrow \Gamma$ is a bijection. In other words, any standard discrete plane can be recoded on a regular lattice (see Section 1).

From now on, let us denote $\mathfrak{P}(a, b, c, \mu)$ the standard discrete plane $\mathfrak{P}(a, b, c, \mu,|a|+|b|+|c|)$. We call unit cube any translate of the fundamental unit cube with integral vertices, that is, any set $(x, y, z)+\mathcal{C}$ where $(x, y, z) \in \mathbb{Z}^{3}$ and $\mathcal{C}$ is the fundamental unit cube (see Figure 2(a)):

$$
\mathcal{C}=\left\{\lambda_{1} \overrightarrow{e_{1}}+\lambda_{3} \overrightarrow{e_{3}}+\lambda_{3} \overrightarrow{e_{3}} \mid\left(\lambda_{1}, \lambda_{2}, \lambda_{3}\right) \in[0,1]^{3}\right\}
$$

Let us now define the three basic faces (see Figure 1):

$$
\begin{aligned}
& E_{1}=\left\{\lambda_{2} \overrightarrow{e_{2}}+\lambda_{3} \overrightarrow{e_{3}} \mid\left(\lambda_{2}, \lambda_{3}\right) \in\left[0,1\left[^{2}\right\},\right.\right. \\
& E_{2}=\left\{-\lambda_{1} \overrightarrow{e_{1}}+\lambda_{3} \overrightarrow{e_{3}} \mid\left(\lambda_{1}, \lambda_{3}\right) \in\left[0,1\left[^{2}\right\},\right.\right. \\
& E_{3}=\left\{-\lambda_{1} \overrightarrow{e_{1}}-\lambda_{2} \overrightarrow{e_{2}} \mid\left(\lambda_{1}, \lambda_{2}\right) \in\left[0,1\left[^{2}\right\} .\right.\right.
\end{aligned}
$$

Let $(x, y, z) \in \mathbb{Z}^{3}$. We call pointed face of type $k$ pointed on $(x, y, z)$ the set $(x, y, z)+E_{k}$ with $k \in\{1,2,3\}$. Notice that each face contains exactly one integral point. We call it the distinguished vertex of the face. Let $\mathcal{P}$ be the plane with equation $a x+b y+c z=\mu$ with $(a, b, c) \in \mathbb{R}^{3}$ and $0 \leq a \leq b \leq c$, let $\mathcal{C}_{\mathcal{P}}$ be the union of the unit cubes intersecting the open half-space $a x+b y+c z<\mu$, and let $\mathfrak{P}_{\mathcal{P}}=\overline{\mathcal{C}_{\mathcal{P}}} \backslash \stackrel{\circ}{\mathcal{C}_{\mathcal{P}}}$, where $\overline{\mathcal{C}_{\mathcal{P}}}$ (resp. $\stackrel{\circ}{\mathcal{C}_{\mathcal{P}}}$ ) is the closure (resp. the interior) of the set $\mathcal{C}_{\mathcal{P}}$ in $\mathbb{R}^{3}$, provided with its usual topology. In [ABS04], it is proved that the set $\mathfrak{P}_{\mathcal{P}}$ is partitioned by pointed faces. Moreover, let $\mathcal{V}_{\mathcal{P}}=\mathfrak{P}_{\mathcal{P}} \cap \mathbb{Z}^{3}$ be the set of vertices of $\mathfrak{P}_{\mathcal{P}}$. Then, $\mathcal{V}_{\mathcal{P}}=\mathfrak{P}(a, b, c, \mu)$ (see (1)). From now on, up to the context and if no confusion is possible, we will call discrete plane indifferently $\mathfrak{P}_{\mathcal{P}}$ and $\mathfrak{P}(a, b, c, \mu)$.

In the present paper we introduce a generalization of the concept of standard discrete planes: the $(1,1,1)$-discrete surfaces (see Figure 3 ). Roughly speaking, a 


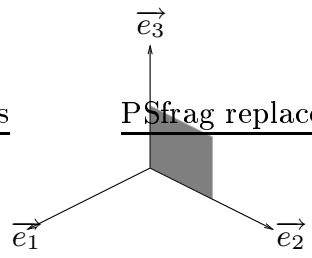

(a) Face of type 1 .

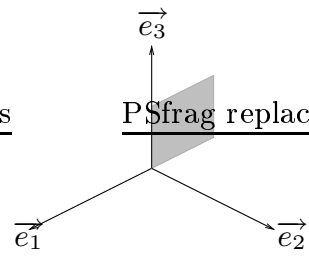

(b) Face of type 2

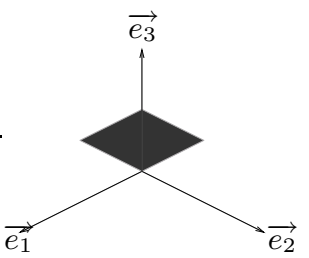

(c) Face of type 3

Fig. 1. The three fundamental faces.

$(1,1,1)$-discrete surface is a subset of $\mathbb{R}^{3}$, partitionable by the pointed faces and in one-to-one correspondence, by the projection map $\pi: \mathbb{R}^{3} \rightarrow\left\{(x, y, z) \in \mathbb{R}^{3}\right.$ $x+y+z=0\}$ with the diagonal plane $\left\{(x, y, z) \in \mathbb{R}^{3} \mid x+y+z=0\right\}$. Then, as performed in the case of the standard discrete planes, given a discrete surface $\mathfrak{S}$, we associate to it a two-dimensional coding depending on the type of the pointed faces partitioning $\mathfrak{S}$. Then, it becomes natural to try to characterize the two-dimensional sequences coding the $(1,1,1)$-discrete surfaces. In other words, given a two-dimensional sequence $U \in\{1,2,3\} \mathbb{Z}^{2}$, does $U$ code a $(1,1,1)$-discrete surface $\mathfrak{S}$ ? Is this problem local? that is, does there exist a finite set of twodimensional finite patterns $\mathcal{E}$ such that: $《 U$ codes a $(1,1,1)$-discrete surface if and only if, for all $\omega \in \mathcal{E}, \omega$ does not belong to the language of $U \gg$ ?

This paper is organized as follows. In Section 1, we define the $(1,1,1)$-discrete surfaces and their two-dimensional codings. In Section 2, after introducing the notions of $\tau$-shape, $\tau$-patterns, $\tau$-complexity and $\tau$-language, we investigate the characterization problem of the sequences $U \in\{1,2,3\} \mathbb{Z}^{2}$ coding discrete surfaces. Then we give the list $\mathfrak{A}$ of permitted $\tau$-patterns (see Figure 4 ), and prove:

Theorem 1. Let $U \in\{1,2,3\}^{\mathbb{Z}^{2}}$. Then $U$ codes a $(1,1,1)$-discrete surface if and only if $\mathcal{L}_{\tau}(u) \subseteq \mathfrak{A}$, where $\mathcal{L}_{\tau}(U)$ is the subset of subwords of $U$ of shape $\tau$.

In Section 3, we show that the standard discrete planes have a canonical structure of $(1,1,1)$-discrete surface and the language of their two-dimensional codings is completely defined by their normal vector and does not depend on their translation parameter. Next, we prove that the $\tau$-complexity of a standard discrete planes is bounded by 6 and equal to 6 for the standard discrete planes with a $\mathbb{Q}$ free normal vector. Finally, in Section 4, we give a first attempt to generalize the study of the dual space of parameters and its corresponding Farey tessellation [VC00]. 


\section{1 (1, 1, 1)-discrete surfaces and two-dimensional codings}

In this section, we introduce the $(1,1,1)$-discrete surfaces and we show how we can recode each discrete surface on a regular lattice.

Let $\pi: \mathbb{R}^{3} \longrightarrow\left\{(x, y, z) \in \mathbb{R}^{3} \mid x+y+z=0\right\}$ be the affine projection along the vector $(1,1,1)$. Then, $\pi$ is explicitly defined by:

$$
\begin{aligned}
\pi: \mathbb{R}^{3} & \longrightarrow\left\{(x, y, z) \in \mathbb{R}^{3} \mid x+y+z=0\right\} \\
(x, y, z) & \mapsto(x-z) \pi\left(\overrightarrow{e_{1}}\right)+(y-z) \pi\left(\overrightarrow{e_{2}}\right) .
\end{aligned}
$$

Let us recall [BV00b,ABS04] that each standard discrete plane is in one-to-

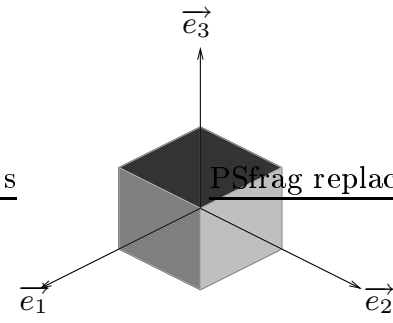

(a) The fundamental unit cube

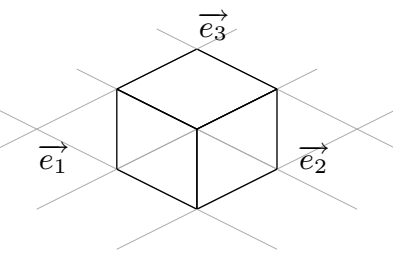

(b) Its projection by $\pi$

Fig. 2. The projection of the fundamental unit cube

one correspondence with the regular lattice $\Gamma=\mathbb{Z} \pi\left(\overrightarrow{e_{1}}\right)+\mathbb{Z} \pi\left(\overrightarrow{e_{2}}\right)=\pi\left(\mathbb{Z}^{3}\right)$ and is partitioned by integral translates of the three basic faces $E_{1}, E_{2}$ and $E_{3}$. Using these properties of standard discrete planes, we define the $(1,1,1)$-discrete surfaces as follows:

Definition 1 ((1,1,1)-discrete surface). Let $\mathfrak{S} \subseteq \mathbb{R}^{3}$. Then $\mathfrak{S}$ is said to be $a(1,1,1)$-discrete surface (or just a discrete surface) if the following conditions hold:

i) the projection map $\pi: \mathfrak{S} \rightarrow\left\{(x, y, z) \in \mathbb{R}^{3} \mid x+y+z=0\right\}$ is a bijection;

ii) $\mathfrak{S}$ is partitioned by pointed faces.

Even if, unfortunately, the terminology can be ambiguous, in particular for the ones who are accustomed with [Fra95,KI00,Mal97,RKW91], we will use the terminology discrete surface instead of $(1,1,1)$-discrete surface in the present paper, in order to simplify notations.

Since the plane $x+y+z=0$ is a disjoint union of a countable set of translates of the tiles $\pi\left(E_{1}\right), \pi\left(E_{2}\right)$ and $\pi\left(E_{3}\right)$, then there exist two sequences $\left(x_{n}, y_{n}, z_{n}\right)_{n \in \mathbb{N}} \in\left(\mathbb{Z}^{3}\right)^{\mathbb{N}}$ and $\left(i_{n}\right)_{n \in \mathbb{N}} \in\{1,2,3\}^{\mathbb{N}}$ such that $\mathfrak{S}=$ $\bigcup_{n \in \mathbb{N}}\left(x_{n}, y_{n}, z_{n}\right)+E_{i_{n}}$. 

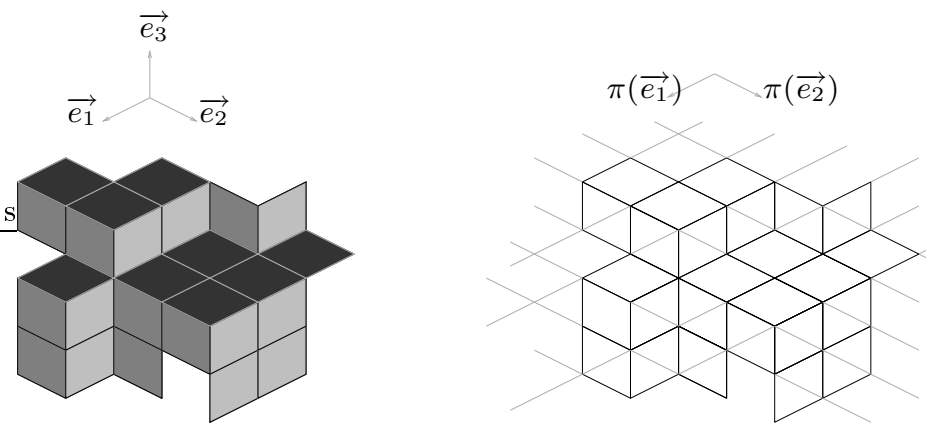

Fig. 3. A piece of a discrete surface and its projection under $\pi$.

A first property of discrete surfaces is that, given $n \in \mathbb{N}$, the point $\left(x_{n}, y_{n}, z_{n}\right)$ cannot have two different types. Moreover, the projection map $\pi: \mathbb{R}^{3} \rightarrow$ $\left\{(x, y, z) \in \mathbb{R}^{3} \mid x+y+z=0\right\}$ provides a one-to-one correspondence between $\left\{\left(x_{n}, y_{n}, z_{n}\right) \mid n \in \mathbb{N}\right\}$ and $\Gamma$. More precisely,

Lemma 1. Let $\mathfrak{S}=\bigcup_{n \in \mathbb{N}}\left(x_{n}, y_{n}, z_{n}\right)+E_{i_{n}}$ be a discrete surface. Then, the following assertions hold:

i) $\forall(m, n) \in \mathbb{N}^{2},\left(x_{m}, y_{m}, z_{m}\right)=\left(x_{n}, y_{n}, z_{n}\right)$ implies $i_{n}=i_{m}$;

ii) the function $\pi:\left\{\left(x_{n}, y_{n}, z_{n}\right) \mid n \in \mathbb{N}\right\} \longrightarrow \mathbb{Z} \pi\left(\overrightarrow{e_{1}}\right)+\mathbb{Z} \overrightarrow{e_{2}}$ is a bijection. In other words, $\left\{\left(x_{n}-z_{n}, y_{n}-z_{n}\right) \mid n \in \mathbb{N}\right\}=\mathbb{Z}^{2}$.

In the present paper, we suppose that the representation of a discrete surface $\mathfrak{S}$ is reduced, that is, Lemma 1 i) and ii) are assumed to hold, and we denote by $\mathcal{V}_{\mathfrak{S}}=\mathfrak{S} \cap \mathbb{Z}^{3}$ the set of vertices of $\mathfrak{S}$.

Since every vertex of $\mathfrak{S}$ has a unique type, then, to each $(m, n) \in \mathbb{Z}^{2}$, we can associate the type of the antecedent $(x, y, z) \in \mathfrak{S}$ of the element $m \pi\left(\overrightarrow{e_{1}}\right)+n \overrightarrow{e_{2}} \in$ $\Gamma$. Thus, we obtain the two-dimensional coding of $\mathfrak{S}$ as follows:

Definition 2 (Two-dimensional coding). Let $\mathfrak{S}=\bigcup_{n \in \mathbb{N}}\left(x_{n}, y_{n}, z_{n}\right)+E_{i_{n}}$ be a discrete surface. The two-dimensional coding of $\mathfrak{S}$ is the sequence $U \in$ $\{1,2,3\}^{\mathbb{Z}^{2}}$ defined as follows:

$$
\forall n \in \mathbb{N}, U_{x_{n}-z_{n}, y_{n}-z_{n}}=i_{n} .
$$

Since we have a two-dimensional coding over the three-letter alphabet $\{1,2,3\}$ of each discrete surface, it becomes natural to investigate the language of these sequences and to study the characterization problem of such a sequence, that is, given a two-dimensional sequence $U \in\{1,2,3\} \mathbb{Z}^{2}$, does it code a discrete surface $\mathfrak{S}$ ? In the next section, we prove that the language of a discrete surface coding is of finite type and we provide the set of permitted patterns.

For every $(m, n) \in \mathbb{Z}^{2}$, let $\tau_{m, n}=\{(m, n),(m, n+1),(m+1, n+1)\}$. A $\tau$-pattern is a pattern with shape $\bar{\tau}$. Hence, following the definitions above, one can define the $\tau$-language and the $\tau$-complexity of a two-dimensional sequence. 


\section{Characterization of the two-dimensional coding of the discrete surfaces}

\subsection{Basic notions on two-dimensional sequences over a finite alphabet}

In this section, we recall some basic notions and terminology concerning the two-dimensional sequences over a finite alphabet.

Let $\sim$ be the equivalence relation over the set $\mathfrak{P}\left(\mathbb{Z}^{2}\right)$ of the finite subsets of $\mathbb{Z}^{2}$, as follows:

$$
\forall\left(\Omega, \Omega^{\prime}\right) \in \mathfrak{P}\left(\mathbb{Z}^{2}\right)^{2}, \quad \Omega \sim \Omega^{\prime} \Longleftrightarrow \exists\left(v_{1}, v_{2}\right) \in \mathbb{Z}^{2}, \Omega=\Omega^{\prime}+\left(v_{1}, v_{2}\right) .
$$

An element $\bar{\Omega}$ of $\mathfrak{P}\left(\mathbb{Z}^{2}\right) / \sim$ is said to be a shape.

Let $\mathcal{A}$ be a finite alphabet. Let $\Omega$ be a finite subset of $\mathbb{Z}^{2}$. A function $\omega: \Omega \rightarrow$ $\mathcal{A}$ is called a finite pointed pattern over the alphabet $\mathcal{A}$. The equivalence relation defined above provides an equivalence relation over the set of the finite pointed patterns over the alphabet $\mathcal{A}$, also denoted $\sim$, as follows: $\forall\left(\omega, \omega^{\prime}\right) \in \mathcal{W}_{\mathcal{A}}^{2}, \omega \sim \omega^{\prime}$ if and only if

$$
\exists\left(v_{1}, v_{2}\right) \in \mathbb{Z}^{2}, \Omega=\Omega^{\prime}+\left(v_{1}, v_{2}\right) \text { and } \forall(m, n) \in \Omega, \omega_{m, n}=\omega_{m+v_{1}, n+v_{2}}^{\prime} .
$$

Let us notice, that given two finite pointed patterns over the alphabet $\mathcal{A}, \omega$ : $\Omega \rightarrow \mathcal{A}$ and $\omega^{\prime}: \Omega^{\prime} \rightarrow \mathcal{A}$, one has $\omega \sim \omega^{\prime}$ implies that $\Omega \sim \Omega^{\prime}$. The equivalence class $\bar{\omega}$ of $\omega$ is said to be a pattern of shape $\bar{\Omega}$. In order to simplify the notation, when no confusion is possible, we will use $\omega(\operatorname{resp} . \Omega)$ instead of $\bar{\omega}(\operatorname{resp} . \bar{\Omega})$.

Let $U \in \mathcal{A}^{\mathbb{Z}^{2}}$ be a two-dimensional sequence and let $\omega: \Omega \rightarrow \mathcal{A}$ be a pattern of shape $\Omega$. An occurrence of $\omega$ in $U$ is an element $\left(m_{0}, n_{0}\right) \in \mathbb{Z}^{2}$ such that for all $(m, n) \in \Omega, \omega_{m, n}=U_{m_{0}+m, n_{0}+n}$. The set of patterns occurring in $U$ is called the language of $U$ and is denoted $\mathcal{L}(U)$. Given a shape $\Omega$, the set of patterns with shape $\Omega$ occurring in $U$ is called the $\Omega$-language of $U$ and is denoted by $\mathcal{L}_{\Omega}(U)$.

Let $\Omega$ be a shape. The $\Omega$-complexity map is the function $p_{\Omega}: \mathcal{A}^{\mathbb{Z}^{2}} \longrightarrow$ $\mathbb{N} \cup\{\infty\}$ defined as follows:

$$
\begin{aligned}
p_{\Omega}: \mathcal{A}^{\mathbb{Z}^{2}} & \longrightarrow \mathbb{N} \cup\{\infty\} \\
U & \mapsto\left|\mathcal{L}_{\Omega}(U)\right|,
\end{aligned}
$$

where $\left|\mathcal{L}_{\Omega}(U)\right|$ is the cardinality of the set $\mathcal{L}_{\Omega}(U)$.

\subsection{Characterization of the two-dimensional coding of a discrete surface}

Let us first reduce the characterization problem to a two-dimensional tiling problem of the plane $\left\{(x, y, z) \in \mathbb{R}^{3} \mid x+y+z=0\right\}$. Indeed, a direct consequence of Definitions 1 and 2 is: 
Lemma 2. Let $U \in\{1,2,3\}^{\mathbb{Z}^{2}}$ be a two-dimensional sequence. The following assertions are equivalent:

i) the set $\mathfrak{S}=\bigcup_{(m, n) \in \mathbb{Z}^{2}}\left\{m \overrightarrow{e_{1}}+n \overrightarrow{e_{2}}+E_{U_{m, n}}\right\}$ is a discrete surface;

ii) the sequence $U$ codes a discrete surface;

iii) the set $\left\{m \pi\left(\overrightarrow{e_{1}}\right)+n \pi\left(\overrightarrow{e_{2}}\right)+\pi\left(E_{U_{m, n}}\right) \mid(m, n) \in \mathbb{Z}^{2}\right\}$ is a partition of the plane $x+y+z=0$.

Let $\mathcal{P}_{0}=\mathbb{R} \pi\left(\overrightarrow{e_{1}}\right)+\mathbb{R} \pi\left(\overrightarrow{e_{2}}\right)$ be the two-dimensional $\mathbb{R}$-vector space of basis $\left\{\pi\left(\overrightarrow{e_{1}}\right), \pi\left(\overrightarrow{e_{2}}\right)\right\}$. Let $|\cdot|_{\infty}: \mathcal{P}_{0} \longrightarrow \mathbb{R}_{+}$be the norm on $\mathcal{P}_{0}$ defined by:

$$
\forall(x, y) \in\left(\mathbb{R}^{2}\right)^{2},\left|x \pi\left(\overrightarrow{e_{1}}\right)+y \pi\left(\overrightarrow{e_{2}}\right)\right|_{\infty}=\max \{|x|,|y|\} .
$$

Let $d_{\infty}$ be the distance on $\mathcal{P}_{0}$ associated to the norm $|\cdot|_{\infty}$, that is,

$$
\forall\left(z, z^{\prime}\right) \in \mathcal{P}_{0}^{2}, d_{\infty}\left(z, z^{\prime}\right)=\left|z-z^{\prime}\right|_{\infty} .
$$

The following lemma is immediate (see Figure 1):

Lemma 3. Let $z, z^{\prime} \in \Gamma=\pi(\mathfrak{S}), z^{\prime \prime} \in \mathcal{P}_{0}$ and $\left(i, i^{\prime}\right) \in\{1,2,3\}^{2}$. Then,

i) $z+\pi\left(E_{i}\right) \cap z^{\prime}+\pi\left(E_{i^{\prime}}\right) \neq \emptyset \Longrightarrow d_{\infty}\left(z, z^{\prime}\right) \leq 1$;

ii) $z^{\prime \prime} \in z+\pi\left(E_{i}\right) \Longrightarrow d_{\infty}\left(z, z^{\prime \prime}\right)<2$.

An interesting consequence of Lemma 3 is that, given a two-dimensional sequence $U \in\{1,2,3\}^{\mathbb{Z}^{2}}$, deciding whether $U$ codes a discrete surface is a local problem. Now, it remains to exhibit a set $\mathfrak{A}$ of permitted patterns.

Roughly speaking, the characterization problem can be divided in two parts: an «injection problem » and a « surjection problem ». The « injection problem » consists in deciding whether a given union of projections of pointed faces is disjoint. The «surjection problem » consists in deciding whether a given union of projections of pointed faces covers $\mathcal{P}_{0}$.

Then, let us first investigate the «injection problem ».

Lemma 4. Let $U \in\{1,2,3\}^{\mathbb{Z}^{2}}$ be a two-dimensional sequence. The following assertions are equivalent:

i) The sets $m \pi\left(\overrightarrow{e_{1}}\right)+n \pi\left(\overrightarrow{e_{2}}\right)+\pi\left(E_{U_{m, n}}\right.$, with $(m, n) \in \mathbb{Z}^{2}$ are relatively disjoint.

ii) For every $(m, n) \in \mathbb{Z}^{2}$, the sets $m^{\prime} \pi\left(\overrightarrow{e_{1}}\right)+n^{\prime} \pi\left(\overrightarrow{e_{2}}\right)+\pi\left(E_{U_{m, n}}\right.$, with $\left(m^{\prime}, n^{\prime}\right) \in$ $\tau_{m, n}$ are relatively disjoint.

Hence, we have obtained a necessary and sufficient condition to decide whether a union of projections of pointed faces is a disjoint union. It remains to find a similar condition for the «surjection problem ». Since the characterization problem is local, a disjoint union of projections of pointed faces will cover the plane $\mathcal{P}_{0}=\left\{(x, y, z) \in \mathbb{R}^{3} \mid x+y+z=0\right\}$ if and only if each point $z$ of $\mathcal{P}_{0}$ will be covered by the projection of a pointed face close to $x$. This is a direct consequence of Lemma 3. Consequently, given a point $g=m \pi\left(\overrightarrow{e_{1}}\right)+n \pi\left(\overrightarrow{e_{2}}\right) \in \Gamma$, a union $\bigcup_{(m, n) \in \mathbb{Z}^{2}} m \pi\left(\overrightarrow{e_{1}}\right)+n \pi\left(\overrightarrow{e_{2}}\right)+\pi\left(E_{U_{m, n}}\right)$ of projections of pointed faces will cover 
$\mathcal{P}_{0}$ if and only if $g+\pi\left(E_{3}\right) \subset \bigcup_{\substack{z=\left(z_{1}, z_{2}\right) \in \Gamma \\ d_{\infty}(z, g)<2}} z_{1} \pi\left(\overrightarrow{e_{1}}\right)+z_{2} \pi\left(\overrightarrow{e_{2}}\right)+\pi\left(E_{U_{z_{1}, z_{2}}}\right)$. In fact, this problem can be reduced to the study of the $\tau$-patterns.

Lemma 5. Let $U \in\{1,2,3\}^{\mathbb{Z}^{2}}$ be a two-dimensional sequence. The following assertions are equivalent:

i) for every $\left(m_{0}, n_{0}\right) \in \mathbb{Z}^{2}$,

$$
\left(m_{0}+1\right) \pi\left(\overrightarrow{e_{1}}\right)+n_{0} \pi\left(\overrightarrow{e_{2}}\right)+\pi\left(E_{3}\right) \subseteq \bigcup_{(m, n) \in \tau_{m_{0}, n_{0}}} m \pi\left(\overrightarrow{e_{1}}\right)+n \pi\left(\overrightarrow{e_{2}}\right)+\pi\left(E_{U_{m, n}}\right) .
$$

ii) $\bigcup_{(m, n) \in \mathbb{Z}^{2}} m \pi\left(\overrightarrow{e_{1}}\right)+n \pi\left(\overrightarrow{e_{2}}\right)+\pi\left(E_{U_{m, n}}\right)=\mathcal{P}_{0}$.

A simple enumeration gives the permitted $\tau$-patterns (see Figure 4 ). In fact,
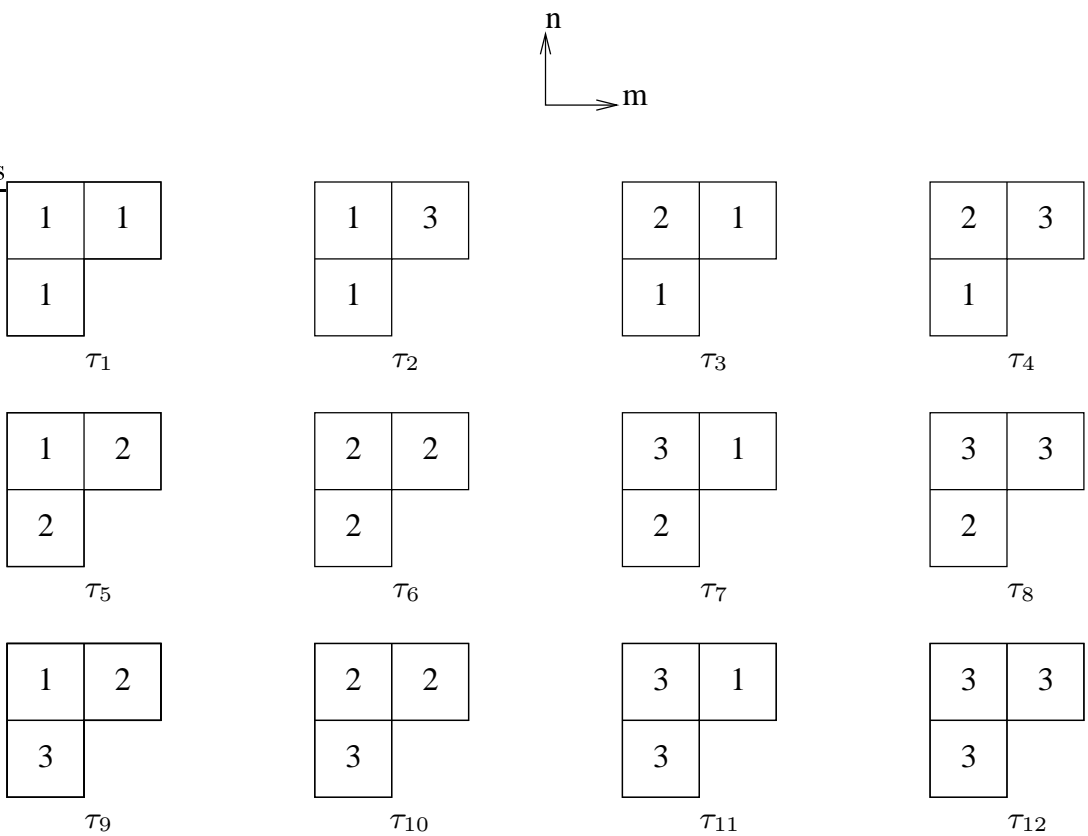

\begin{tabular}{|l|l|}
\hline 3 & 1 \\
\hline 3 & \multicolumn{1}{|r|}{1} \\
\hline \multicolumn{2}{|c|}{$\tau_{11}$}
\end{tabular}

\begin{tabular}{|l|l|}
\hline 3 & 3 \\
\hline 3 & \\
\hline & \\
$\tau_{12}$
\end{tabular}

Fig. 4. The set $\mathfrak{A}$ of permitted $\tau$-patterns of a discrete surface

we have proved that:

Theorem 2. Let $\mathfrak{A}$ be the set of allowed $\tau$-patterns (see Figure 4). Let $U \in$ $\{1,2,3\}^{\mathbb{Z}^{2}}$ be a two-dimensional sequence over the three-letter alphabet $\{1,2,3\}$. Then $U$ codes a discrete surface $\mathfrak{S}$ if and only if $\mathcal{L}_{\tau}(U) \subseteq \mathfrak{A}$. 


\section{A particular case of discrete surfaces: the standard discrete planes}

In this section, we investigate the standard discrete planes with a positive normal vector and show that they admit a canonical structure of discrete surface. From now on, we suppose that $(a, b, c) \in \mathbb{R}_{+}^{3}$.

\subsection{Preliminaries}

For the moment, we have defined the discrete surfaces via a one-to-one condition on the projection map $\pi: \mathfrak{S} \longrightarrow\left\{(x, y, z) \in \mathbb{R}^{3} \mid x+y+z=0\right\}$. In [BV00b,ABS04], it is proved that a standard discrete plane is in bijection with $\Gamma=\pi\left(\mathbb{Z}^{3}\right)$. Let $\mathcal{P}$ be a plane with equation $a x+b y+c z=\mu$. To prove that $\mathfrak{P}_{\mathcal{P}}$ (see Section 1) is a discrete surface, we have to show that $\pi: \mathfrak{P}_{\mathcal{P}} \longrightarrow\left\{(x, y, z) \in \mathbb{R}^{3} \mid x+y+z=0\right\}$ is a bijection, or equivalently, that the coding of $\mathfrak{P}_{\mathcal{P}}$ codes a discrete surface. Let us recall how to build the two-dimensional coding of $\mathfrak{P}_{\mathcal{P}}$. It is based on Lemma 6 .

Lemma 6. [ABS04] Let $(x, y, z) \in \mathcal{V}_{\mathcal{P}}$ and $k \in\{1,2,3\}$. Let $I_{1}=\left[0, a\left[, I_{2}=\right.\right.$ $\left[a, a+b\left[\right.\right.$ and $I_{3}=[a+b, a+b+c[$. Then, the following assertions are equivalent:

i) the point $(x, y, z)$ is of type $k$, that is, $(x, y, z)+E_{k} \subseteq \mathfrak{P}_{\mathcal{P}}$;

ii) $a x+b y+c z-\mu \in I_{k}$;

iii) $a(x-z)+b(y-z)-\mu \bmod (a+b+c) \in I_{k}$.

The two-dimensional sequence $U$ coding $\mathfrak{P}_{\mathcal{P}}$ is defined as follows:

$$
\forall(m, n) \in \mathbb{Z}^{2}, \forall k \in\{1,2,3\}, U_{m, n}=k \Longleftrightarrow a m+b n-\mu \in I_{k} .
$$

The discrete surface structure of $\mathfrak{P}_{\mathcal{P}}$ follows from:

Theorem 3. The set $\mathfrak{P}_{\mathcal{P}}$ is a discrete surface.

Proof. Let $U$ be the two-dimensional coding of $\mathfrak{P}_{\mathcal{P}}$. Let us show that $U$ codes a discrete surface. Indeed, since $U$ codes $\mathfrak{P}_{\mathcal{P}}$, we will deduce that $\mathfrak{P}_{\mathcal{P}}$ is a discrete surface. Let $k \in\{1,2,3\}$ and let us consider a $\tau$-pattern $\omega$ such that $\omega_{0,0}=1$. Let $(m, n) \in \mathbb{Z}^{2}$ be an occurrence of $\omega$, that is, $U_{m+i, n+j}=\omega_{k i, j}$ for $(i, j) \in$ $\tau_{0,0}$. Let us first suppose that $(m, n)=(0,0)$. Then, we deduce that $\mu \in[0, a[$ $\bmod (a+b+c)$. Hence $\mu+a+b \in[a+b, 2 a+b[\bmod (a+b+c)$. If $a<c$, then $\mu+a+b \in\left[a+b, a+b+c\left[\bmod (a+b+c)\right.\right.$ and $\omega_{1,1}=3$. Conversely, if $a>c$, then $2 a+b \in\left[0, a\left[\bmod (a+b+c)\right.\right.$ and $\omega_{1,1}=1$. In all cases, $\omega_{1,1} \neq 2$. If $(m, n) \neq(0,0)$, we similarly prove that $\omega_{1,1} \in\{1,3\}$. The other forbidden $\tau$-patterns can be excluded in the same way. 


\subsection{Characterization of the language of a standard discrete plane}

In this section, given a standard discrete plane $\mathfrak{P}$, we call language of a standard discrete plane the language of the two-dimensional coding of $\mathfrak{P}$.

Let $(\alpha, \beta) \in \mathbb{R}_{+}^{2}$. The rotation $R_{\alpha}$ of angle $\alpha$ modulo $\beta$ is the function $R_{\alpha}$ : $[0, \beta[\longrightarrow[0, \beta[$ defined as follows:

$$
\begin{aligned}
R_{\alpha}:[0, \beta[ & \longrightarrow \quad[0, \beta[ \\
x & \mapsto x+\alpha \bmod \beta .
\end{aligned}
$$

From now on, $R_{a}$ (resp. $R_{b}$ ) denotes the rotation of angle $a$ (resp. of angle $b$ ) modulo $a+b+c$.

Lemma 7. Let $U \in\{1,2,3\}^{\mathbb{Z}^{2}}$ be the two-dimensional coding of the standard discrete plane $\mathfrak{P}(a, b, c, \mu)$. Let $\omega: \Omega \rightarrow\{1,2,3\}$ be a pattern. Then, the following assertions are equivalent:

i) $\omega \in \mathcal{L}(U)$, that is, there exists $\left(k, k^{\prime}\right) \in \mathbb{Z}^{2}$ such that:

$$
\forall(m, n) \in \Omega, \omega_{m, n}=U_{m+k, n+k^{\prime}}
$$

ii) there exists $\left(k, k^{\prime}\right) \in \mathbb{Z}^{2}$ such that:

$$
a k+b k^{\prime}-\mu \in \bigcap_{(i, j) \in \Omega} R_{a}^{-i} \circ R_{b}^{-j}\left(I_{\omega_{i, j}}\right) .
$$

In [Rév91,And93,VC00], the authors considered standard discrete planes $\mathfrak{P}(a, b, c, \mu)$ with $(a, b, c, \mu) \in \mathbb{Z}^{4}$ and $\operatorname{gcd}(a, b, c)=1$. In [BV00b,Lot02,ABS04], the authors investigated the standard discrete lines or planes with a $\mathbb{Q}$-free normal vector. Let us recall that a $n$-uple $\left(a_{1}, \ldots, a_{n}\right) \in \mathbb{R}^{n}$ is said to be $\mathbb{Q}$-free if for every $\left(x_{1}, \ldots, x_{n}\right) \in \mathbb{Q}^{n}$, one has:

$$
\sum_{i=1}^{n} a_{i} x_{i}=0 \Longleftrightarrow \forall i \in \llbracket 1, n \rrbracket, x_{i}=0 .
$$

In fact, this two-case division is not necessary to study the language of the two-dimensional coding of a standard discrete plane. More precisely:

Corollary 1. Let $U \in\{1,2,3\}$ be the two-dimensional coding of the standard discrete plane $\mathfrak{P}(a, b, c, \mu)$. Let $\omega$ be an $\Omega$-pattern. Then,

$$
\omega \in \mathcal{L}(U) \Longleftrightarrow I_{\omega}=\bigcap_{(i, j) \in \Omega} R_{a}^{-i} \circ R_{b}^{-j}\left(I_{\omega_{i, j}}\right) \neq \emptyset .
$$

A direct consequence of Corollary 1 is:

Corollary 2. Let $U \in\{1,2,3\}^{\mathbb{Z}^{2}}$ (resp. $U^{\prime} \in\{1,2,3\}^{\mathbb{Z}^{2}}$ ) be the two-dimensional coding of the standard discrete plane $\mathfrak{P}(a, b, c, \mu)$ (resp. $\left.\mathfrak{P}\left(a^{\prime}, b^{\prime}, c^{\prime}, \mu^{\prime}\right)\right)$. Let us suppose that $\mathfrak{P}$ is parallel to $\mathfrak{P}^{\prime}$, that is, there exits $\alpha \in \mathbb{R}$ such that $(a, b, c)=$ $\alpha\left(a^{\prime}, b^{\prime}, c^{\prime}\right)$. Then $\mathcal{L}(U)=\mathcal{L}\left(U^{\prime}\right)$. 
Since two sequences coding two parallel standard discrete planes have the same language, it becomes natural to investigate the following problem: given two standard discrete planes $\mathfrak{P}=\mathfrak{P}(a, b, c, \mu)$ and $\mathfrak{P}^{\prime}=\mathfrak{P}\left(a^{\prime}, b^{\prime}, c^{\prime}, \mu^{\prime}\right)$ and given $U \in\{1,2,3\}^{\mathbb{Z}^{2}}$ (resp. $U^{\prime} \in\{1,2,3\} \mathbb{Z}^{2}$ ) the two-dimensional coding of $\mathfrak{P}$ (resp. $\left.\mathfrak{P}^{\prime}\right)$. Let us suppose that $\mathcal{L}(U)=\mathcal{L}\left(U^{\prime}\right)$. Are the standard discrete planes $\mathfrak{P}$ and $\mathfrak{P}^{\prime}$ parallel? The answer is given by the following theorem:

Theorem 4. Let $(a, b, c, \mu) \in \mathbb{Z}^{4}$ (resp. $\left.\left(a^{\prime}, b^{\prime}, c^{\prime}, \mu^{\prime}\right) \in \mathbb{Z}^{4}\right)$. Let $U \in\{1,2,3\} \mathbb{Z}^{2}$ (resp. $U^{\prime} \in\{1,2,3\}^{\mathbb{Z}^{2}}$ ) be the two-dimensional coding of the standard discrete plane $\mathfrak{P}=\mathfrak{P}(a, b, c, \mu)$ (resp. $\left.\mathfrak{P}^{\prime}=\mathfrak{P}\left(a^{\prime}, b^{\prime}, c^{\prime}, \mu^{\prime}\right)\right)$. Then, the following assertions are equivalent:

i) the planes $a x+b y+c z=\mu$ and $a^{\prime} x+b^{\prime} y+c^{\prime} z=\mu^{\prime}$ are parallel;

ii) there exists $\left(m_{0}, n_{0}\right) \in \mathbb{Z}^{2}$ such that, for every $(m, n) \in \mathbb{Z}^{2}, U_{m, n}=$ $U_{m+m_{0}, n+n_{0}}^{\prime}$

iii) $\mathcal{L}(U)=\mathcal{L}\left(U^{\prime}\right)$.

Proof. Let us first prove that given a square $S$ of edge $a+b+c$, the number of $a$ (resp. $b, c)$ in a subwords $\omega: S \rightarrow\{1,2,3\}$ is $a(a+b+c)$ (resp. $b(a+b+c)$, $c(a+b+c))$. In fact, it is sufficient to study the case $S=\llbracket 0, a+b+c-1 \rrbracket^{2}$. The general case is a direct consequence of Corollary 1 .

Let us assume that $\operatorname{gcd}(a, b, c)=1$. Then, $\operatorname{gcd}(a, b, a+b+c)=1$. Hence, for every element $k \in \llbracket 0, a-1 \rrbracket$, there exists $(x, y) \in \mathbb{Z}^{2}$ such that $a x+b y-\mu \equiv k$ $\bmod a+b+c$. Let $(m, n) \in \llbracket 0, a+b+c-1 \rrbracket^{2}$ such that $m \equiv x \bmod a+b+c$ and $n \equiv y \bmod a+b+c$. Then $a m+b n-\mu \equiv a x+b x-\mu \equiv x \bmod (a+b+c)$ and $U_{m, n}=1$. Let $k \in \llbracket 0, a+b+c-1 \rrbracket$. Then $U_{m-k b, n+k a}=1$. Moreover, for all $(m, n) \in \llbracket 0, a+b+c-1 \rrbracket^{2},(m, n) \equiv(m-k b, n+k a) \bmod a+b+c$ if and only if $k=0$. Indeed, let us suppose that $k a \equiv k b \equiv 0 \bmod a+b+c$ and let $(u, v) \in \mathbb{Z}^{2}$ such that $a u+b v \equiv 1 \bmod a+b+c$. Then $k \equiv k(a u+b v) \equiv$ $k a u+k a v \equiv 0 \bmod a+b+c$. Since $k \in \llbracket 0, a+b+c-1 \rrbracket$, we deduce that $k=0$. Hence $|U|_{a} \geq a(a+b+c)$. We similarly prove that $|U|_{b} \geq b(a+b+c)$ and $|U|_{c} \geq c(a+b+c)$. Finally, since $|U|_{a}+|U|_{b}+|U|_{c} \geq(a+b+c)^{2}$, one has the desired result. If $\operatorname{gcd}(a, b, c)=d$, then let us define $a^{\prime}=a / d, b^{\prime}=b / d$ and $c^{\prime}=c / d$. Then, let us denote $|\widetilde{U}|_{a}$ (resp. $\left.|\widetilde{U}|_{b},|\widetilde{U}|_{c}\right)$ the number of $a$ (resp. $b$, $c)$ in the square $\left.S=\llbracket 0, \frac{a+b+c}{d}-1\right]^{2}$. Since $(0, a+b+c)$ and $(a+b+c, 0)$ are two periodic vectors of $U$, that is, for all $\left(k, k^{\prime}\right) \in \mathbb{Z}^{2}$ and for all $(m, n) \in \mathbb{Z}^{2}$, we have $U_{m, n}=U_{m+k(a+b+c), n+k^{\prime}(a+b+c)}$, then $|U|_{a}=d^{2}|\widetilde{U}|_{a}$ (resp. $|U|_{b}=d^{2}|\widetilde{U}|_{b}$, $\left.|U|_{c}=d^{2}|\widetilde{U}|_{c}\right)$. By the same way as above, we obtain that $|\widetilde{U}|_{a}=a \frac{a+b+c}{d^{2}}$ (resp. $|\widetilde{U}|_{b}=b \frac{a+b+c}{d^{2}},|\widetilde{U}|_{c}=c \frac{a+b+c}{d^{2}}$ ) and the desired result follows.

It is sufficient to prove that ii) $\Longrightarrow$ iii). Let us suppose that $\mathcal{L}(U)=\mathcal{L}\left(U^{\prime}\right)$. Let $k=\operatorname{lcm}\left(a+b+c, a^{\prime}+b^{\prime}+c^{\prime}\right) /(a+b+c)$ and $k^{\prime}=\operatorname{lcm}\left(a+b+c, a^{\prime}+b^{\prime}+c^{\prime}\right) /\left(a^{\prime}+\right.$ $\left.b^{\prime}+c^{\prime}\right)$. Let $\left(a_{1}, b_{1}, c_{1}, \mu_{1}\right)=k(a, b, c, \mu)$ and $\left(a_{1}^{\prime}, b_{1}^{\prime}, c_{1}^{\prime}, \mu_{1}^{\prime}\right)=k^{\prime}\left(a^{\prime}, b^{\prime}, c^{\prime}, \mu^{\prime}\right)$. Let $V \in\{1,2,3\}^{\mathbb{Z}^{2}}$ (resp. $V^{\prime} \in\{1,2,3\}^{\mathbb{Z}^{2}}$ ) be the two-dimensional sequence coding the standard discrete plane $\mathfrak{P}\left(a_{1}, b_{1}, c_{1}, \mu_{1}\right)$ (resp. $\left.\mathfrak{P}\left(a_{1}^{\prime}, b_{1}^{\prime}, c_{1}^{\prime}, \mu_{1}^{\prime}\right)\right)$. Then, $V=U$ and $V^{\prime}=U^{\prime}$. Since $a_{1}+b_{1}+c_{1}=a_{1}^{\prime}+b_{1}^{\prime}+c_{1}^{\prime}$ and one has $|V|_{a}=\left|V^{\prime}\right|_{a}$ (resp. $\left.|V|_{b}=\left|V^{\prime}\right|_{b},|V|_{c}=\left|V^{\prime}\right|_{c}\right)$ ) (see Lemma 7), we have $a_{1}\left(a_{1}+b_{1}+c_{1}\right)=$ 
$a_{1}^{\prime}\left(a_{1}+b_{1}+c_{1}\right)\left(\right.$ resp. $b_{1}\left(a_{1}+b_{1}+c_{1}\right)=b_{1}^{\prime}\left(a_{1}+b_{1}+c_{1}\right), c_{1}\left(a_{1}+b_{1}+c_{1}\right)=$ $\left.c_{1}^{\prime}\left(a_{1}+b_{1}+c_{1}\right)\right)$, that is, $k a=k^{\prime} a^{\prime}, k b=k^{\prime} b^{\prime}$ and $k c=k^{\prime} c^{\prime}$.

\section{An analytic description of the $\tau$-language of the standard discrete planes}

In Theorem 4, we proved that the language of a standard discrete plane with a positive normal vector does not depend on its translation parameter $\mu$ and is completely defined by its normal vector $(a, b, c)$.

In this section, we provide an analytic way to describe the $\tau$-language $\mathcal{L}_{\tau}(\mathfrak{P})$ of a standard discrete plane $\mathfrak{P}$. This kind of investigation can be compared to [Col02,Gér99,VC99,Vui98]

Roughly speaking, to each $\tau$-pattern $\omega$ of $\mathfrak{A}$, we associate the subset of the triples $(a, b, c)$ of $\mathbb{R}^{3}$, such that $\omega$ has an occurrence in the two-dimensional coding of any standard discrete planes with normal vector $(a, b, c)$.

Let us recall that we can defined the discrete surface structure of a standard discrete plane if and only if its normal vector $(a, b, c)$ is positive, that is $\min \{a, b, c\} \geq 0$. In the present section, if it is not mentioned, we will suppose $a, b, c$ to be positive and $c \neq 0$.

Since it is easily checked that $\mathfrak{P}(a, b, c, \mu)=\mathfrak{P}(a / c, b / c, 1, \mu / c)$, let us assume that $c=1$. Hence, to each $\tau$-pattern $\omega$ of $\mathfrak{A}$, we will associate the subset of the pairs $(a, b)$ of $\mathbb{R}^{2}$, such that $\omega$ has an occurrence in the two-dimensional coding of any standard discrete planes with normal vector $(a, b, 1)$.

For instance, let us consider the following $\tau$-patterns:

$$
\omega=\begin{aligned}
& 12 \\
& 3
\end{aligned}
$$

Then, following Corollary 1, one has:

$$
\begin{aligned}
\omega \in \mathcal{L}_{\tau}(\mathfrak{P}) & \Longleftrightarrow I_{3} \cap R_{b}^{-1}\left(I_{1}\right) \cap R_{a}^{-1} \circ R_{b}^{-1}\left(I_{2}\right) \neq \emptyset \\
& \Longleftrightarrow b<a+c .
\end{aligned}
$$

Then, assuming that $c=1$, we associate to $\omega$ the set $\left\{(a, b) \in \mathbb{R}^{2} \mid b<a+1\right\}$, representing the pairs $(a, b, 1) \in \mathbb{R}_{+}^{3}$, such that $\omega$ occurs in the two-dimensional coding of any standard discrete plane with normal vector $(a, b, 1)$.

Considering the $\tau$-patterns of Figure 4, we obtain the following graphical representation (see Figure 5): 


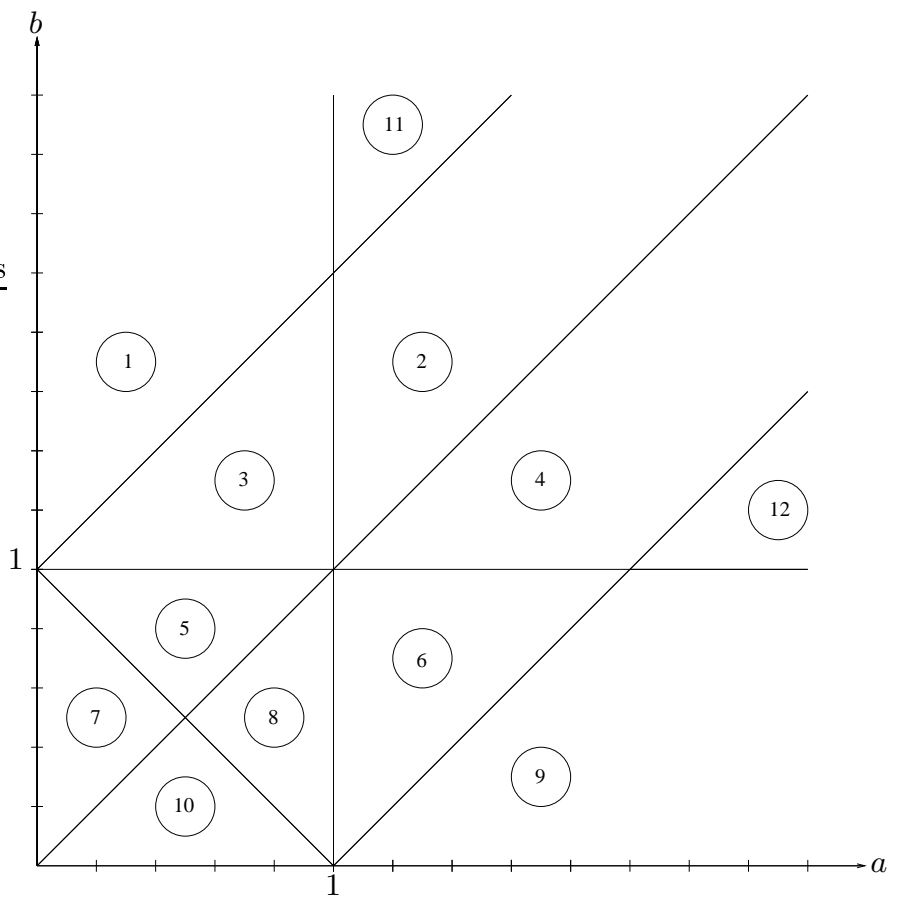

Fig. 5. Graphical representation of the $\tau$-language of the standard discrete planes.

\begin{tabular}{|c||c|c|c|c|c|c|c|c|c|c|c|c|}
\hline$\tau$ zone & $\tau_{1}$ & $\tau_{2}$ & $\tau_{3}$ & $\tau_{4}$ & $\tau_{5}$ & $\tau_{6}$ & $\tau_{7}$ & $\tau_{8}$ & $\tau_{9}$ & $\tau_{10}$ & $\tau_{11}$ & $\tau_{12}$ \\
\hline 1 & & & & $\times$ & $\times$ & $\times$ & $\times$ & $\times$ & & $\times$ & & \\
\hline 2 & & & $\times$ & $\times$ & $\times$ & & $\times$ & & $\times$ & $\times$ & & \\
\hline 3 & & & & $\times$ & $\times$ & & $\times$ & $\times$ & $\times$ & $\times$ & & \\
\hline 4 & & $\times$ & $\times$ & $\times$ & $\times$ & & $\times$ & & $\times$ & & & \\
\hline 5 & & & & $\times$ & & & $\times$ & $\times$ & $\times$ & $\times$ & $\times$ & \\
\hline 6 & & $\times$ & $\times$ & $\times$ & & & $\times$ & & $\times$ & & $\times$ & \\
\hline 7 & & & & $\times$ & & & & $\times$ & $\times$ & $\times$ & $\times$ & $\times$ \\
\hline 8 & & $\times$ & & $\times$ & & & $\times$ & $\times$ & $\times$ & & $\times$ & \\
\hline 9 & $\times$ & $\times$ & $\times$ & & & & $\times$ & & $\times$ & & $\times$ & \\
\hline 10 & & $\times$ & & $\times$ & & & & $\times$ & $\times$ & & $\times$ & $\times$ \\
\hline 11 & & & $\times$ & $\times$ & $\times$ & $\times$ & $\times$ & & & $\times$ & & \\
\hline 12 & $\times$ & $\times$ & $\times$ & & $\times$ & & $\times$ & & $\times$ & & & \\
\hline
\end{tabular}

For every $k \in \llbracket 1,12 \rrbracket$, let $\mathcal{L}_{k}$ be the set of $\mathfrak{A}$-patterns associated to the $k$-th zone of Figure 5. Then, a direct consequence of Corollary 1 and Figure 5 is:

Theorem 5. Let $(a, b) \in \mathbb{N}^{2},\left\{n_{1}, \ldots, n_{k}\right\} \subseteq \llbracket 1,12 \rrbracket$ be the finite set of all the zones of Figure 5 containing $(a, b)$ and $\mathcal{L}(a, b)$ be the language of the standard 
discrete plane with normal vector $(a, b, 1)$. Then,

$$
\mathcal{L}(a, b)=\bigcap_{i=1}^{k} \mathcal{L}_{i} .
$$

Let us call $\tau$-complexity of a standard discrete plane $\mathfrak{P}$ the $\tau$-complexity of the two-dimensional coding of $\mathfrak{P}$. Then, a direct consequence of Figure 5 and Theorem 5 is:

Corollary 3. Let $U \in\{1,2,3\} \mathbb{Z}^{2}$ be the two-dimensional coding of a standard discrete plane with normal vector $(a, b, c) \in \mathbb{N}^{3}$ with $c \neq 0$ and let $\left\{n_{1}, \ldots, n_{k}\right\} \subseteq$ $\llbracket 1,12 \rrbracket$ be the finite set of all the zones of Figure 5 containing $(a / c, b / c)$. Then,

$$
p_{\tau}(U)=6-k+1
$$

Remark 1. Let $U \in\{1,2,3\} \mathbb{Z}^{2}$ be the two-dimensional coding of a standard discrete plane $\mathfrak{P}(a, b, c, \mu)$. One can have $p_{\tau}(U)=6$ with while $\{a, b, c\}$ is non$\mathbb{Q}$-free. For instance, let $a=1, b=3$ and $c=5$ (see Figure 6).
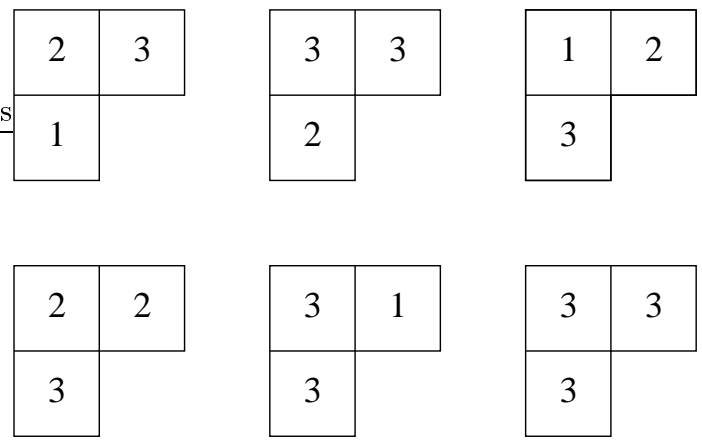

Fig. 6. $\tau$-patterns of the sequence associated to the standard discrete plane $\mathfrak{P}(1,3,5,0)$.

\section{Acknowledgments}

I would like to thank Pierre Arnoux for having pointed me out the recognition problem of combinatorial codings of discrete surfaces and the referees for the useful suggestions they made.

\section{References}

[ABS04] P. Arnoux, V. Berthé, and A. Siegel. Two-dimensional iterated morphisms and discrete planes. Theoretical Computer Science, 319:145-176, 2004. 
[And93] É. Andrès. Le plan discret. Colloque de géométrie discrète en imagerie : fondements et applications, Septembre 1993.

[BCK04] V. Brimkov, D. Coeurjolly, and R. Klette. Digital planarity - a review. Technical Report RR2004-24, Laboratoire LIRIS - Université Lumière Lyon 2, may 2004.

[Buz02] L. Buzer. An incremental linear time algorithm for digital line and plane recognition using a linear incremental feasibility problem. In Proceedings of the 10th International Conference on Discrete Geometry for Computer Imagery, pages 372-381. Springer-Verlag, 2002.

[BT04] V. Berthé and R. Tijdeman. Lattices and multi-dimensional words. Theoretical Computer Science, 319:177-202, 2004.

[BV00a] V. Berthé and L. Vuillon. Suites doubles de basse complexité. Journal de Théorie des Nombres de Bordeaux, 12:179-208, 2000.

[BV00b] V. Berthé and L. Vuillon. Tilings and rotations on the torus: a twodimensional generalization of sturmian sequences. Discrete Mathematics, 223:27-53, 2000.

[Col02] M.A. Jacob-Da Col. About local configurations in arithmetic planes. Theor. Comput. Sci., 283(1):183-201, 2002.

[DRR96] I. Debled-Rennesson and J.P. Réveillès. Incremental algorithm for recognizing pieces of digital planes. In Robert A. Melter, Angela Y. Wu, and Longin Latecki, editors, Vision Geometry V, volume 2826 of SPIE Proceedings, pages 140-151, August 1996.

[Fra95] J. Françon. Discrete combinatorial surfaces. Graph. Models Image Process., $57(1): 20-26,1995$.

[Gér99] Y. Gérard. Local configurations of digital hyperplanes. In Proceedings of the 8th International Conference on Discrete Geometry for Computer Imagery, pages 65-75. Springer-Verlag, 1999.

[KI00] Y. Kenmochi and A. Imiya. Naive planes as discrete combinatorial surfaces. In Proceedings of the 9th International Conference on Discrete Geometry for Computer Imagery, pages 249-261. Springer-Verlag, 2000.

[Lot02] Lothaire. Algebraic Combinatorics on Words. Cambridge University Press, 2002.

[Mal97] R. Malgouyres. A definition of surfaces of $\mathbb{Z}^{3}$. a new 3 d discrete jordan theorem. Theor. Comput. Sci., 186(1-2):1-41, 1997.

[Meg84] N. Megiddo. Linear programming in linear time when the dimension is fixed. J. ACM, 31(1):114-127, 1984.

[PS85] F.P. Preparata and M.I. Shamos. Computational geometry: an introduction. Springer-Verlag New York, Inc., 1985.

[Rév91] J.P. Réveillès. Géométrie discète, calcul en nombres entiers et algorithmique. PhD thesis, Université Louis Pasteur, Strasbourg, 1991.

[RKW91] A. Rosenfeld, T.Y. Kong, and A.Y. Wu. Digital surfaces. CVGIP: Graph. Models Image Process., 53(4):305-312, 1991.

[VC99] J. Vittone and J.M. Chassery. (n, m)-cubes and farey nets for naive planes understanding. In Proceedings of the 8th International Conference on Discrete Geometry for Computer Imagery, pages 76-90. Springer-Verlag, 1999.

[VC00] J. Vittone and J.M. Chassery. Recognition of digital naive planes and polyhedrization. In DGCI: International Workshop on Discrete Geometry for Computer Imagery, pages 297-307, 2000.

[Vui98] L. Vuillon. Combinatoire des motifs d'une suite sturmienne bidimensionnelle. Theor. Comput. Sci., 209(1-2):261-285, 1998. 